\title{
Cross-Cultural adaptation, translation, and Moroccan validation of caregiver Oncology Quality of Life (CarGoQoL) questionnaire
}

\author{
Aitouma Ahlam ${ }^{1,2 *}$, Mrabti Hind ${ }^{1,2}$, Haddou Rahou Bouchra ${ }^{3}$, Razine Rachid4 and Errihani Hassan ${ }^{1,2}$ \\ ${ }^{1}$ Department of Medical Oncology, National Institute of Oncology, Rabat, Morocco \\ ${ }^{2}$ Translational Oncology Research Team, Faculty of Medicine and Pharmacy Mohamed V University, Rabat, Morocco \\ ${ }^{3}$ Research Department, Higher Institute of Nursing Professions and Technical Health, Rabat, Morocco \\ ${ }^{4}$ Laboratory of Social Medicine and Public Health, Faculty of Medicine and Pharmacy, Mohamed V University, Rabat, Morocco
}

\begin{abstract}
Background: The Caregivers Oncology Quality of Life (CarGoQoL) questionnaire is a multidimensional self-administered questionnaire composed of 29 items covering 10 dimensions and has been validated in two versions: French and American. The objective of this study is to validate the Moroccan version of CarGoQoL to measure the caregivers quality of life of palliative cancer patients in Morocco.

Methods: The transcultural adaptation of CarGoQoL was carried out according to the recommendations of the Mapi language translation manual and those of Beaton. The final version obtained was administered to 120 caregivers of cancer patients in the palliative phase over a period of seven months at the National Oncology Institute in Rabat, Morocco. The questionnaire was administered twice with an interval of three to seven days to assess its reproducibility. The statistical analysis first described the study population and then verified the psychometric properties of the scale. Reliability was verified by the Cronbach alpha coefficient and intra-class correlation coefficients. The validity of the scale was measured by the multi-item correlation.
\end{abstract}

Results: The psychometric properties of CarGoQoL were generally good. A Cronbach $\alpha$ coefficient of 0.82 was obtained indicating a good internal consistency except for the private life dimension $(\alpha=0.14)$. A good reliability of the test re-test was also observed, with an item internal consistency of 0.9 . Missing data and ceiling effect were low; some ceiling effects were observed for Relationship with healthcare (38.9\%); correlations were confirmed between the different dimensions that corresponding to the same domain; significant associations were identified for caregiver and patient characteristics.

Conclusions: This study has shown that the Moroccan Arabic dialectal version of the CarGoQoL questionnaire is reliable and valid in measuring the quality of life of caregivers of palliative cancer patients in Morocco.

\begin{abstract}
Abbreviations: CarGoQoL: Care giver oncology quality of life; PsWB: Psychological wellbeing; B: Burden; RHC: Relationship with healthcare; AF: Administration and finances; COP: Coping; PhWB: Physical wellbeing; SE: Self-esteem; LEI: Leisure time; SS: Social support; PL: Private life; ICC: Item internal consistency; IDV: Item discriminant validity; a: Alpha Cronbach's alpha.
\end{abstract}

\section{Background}

Due to the continuing advances in cancer treatment, cancer is likely to increasingly become a chronic disease [1]. Cancer is a catastrophic event for the family and can impose serious stress on patients and their family members [2]. The caregiver accompanies his or her loved one at the time of diagnosis, during treatment, and follows the news about the disease's progression. He is afraid of the future and death which are stressful events [3]. Informal caregivers of patients with a life-threatening disease such as cancer are often deeply involved in the patient's disease and provide extraordinary and uncompensated care. The caregivers may take on a range of disease related task and provide emotional support [4-6].

It is recognized that caregiving has negative effects on the caregiver quality of life (QoL) $[7,8]$. The QoL of informal caregiver is an important issue both in the context of active/curative and palliative/supportive treatment [9].

In order to measure the quality of life of caregiver several scientific studies have created and validated different scales.

Recently, a French team has developed the CarGoQoL questionnaire [10], which has already been validated by a study on caregivers of glioma patients [7] and by a validation study of an American version [11]. It has the advantage of a multidimensional approach and is based exclusively on the caregiver's perspective.

In Morocco, no studies have been conducted on the measurement of health-related quality of life among caregivers of palliative cancer

${ }^{*}$ Correspondence to: Aitouma Ahlam, Department of Medical Oncology, National Institute of Oncology, Rabat, Morocco, Translational Oncology Research Team, Faculty of Medicine and Pharmacy Mohamed V University, Rabat, Morocco, E-mail: Ahlamorim2005@gmail.com

Key words: caregivers, palliative cancer, quality of life, CarGoQoL, reliability, validity

Received: December 27, 2020; Accepted: January 08, 2021; Published: January 11,2021 
patients [12]. The availability of a valid and reliable Moroccan version of quality-of-life measurement instruments is essential to collect such measures. There are several advantages to culturally adapting an existing instrument rather than composing a new one [13].

Most adaptation processes involve two main steps: the assessment of equivalence between the source and adapted instrument and the assessment of the measurement properties of the adapted instrument [12]. The intercultural adaptation of a quality-of-life instrument is a step-by-step process [13-16].

In this context, the objective of this study was to carry out a transcultural adaptation and validity of the CarGoQoL questionnaire to evaluate the psychometric properties of the translated version.

\section{Methods}

\section{Characteristics of the CarGoQoL original version}

For caregivers' characteristics, we collected information on gender, age, marital status, nature of the relationship with the patient and duration of care. For patient we collected information on sex, age, cancer location, disease duration, stage, Karnovsky Index.

The CarGOQoL is a specific questionnaire that includes 29 questions describing 10 dimensions: psychological well-being, burden, relationship to health care, administration and finance, adaptation, physical well-being, self-esteem, leisure, social support and private life. The CarGOQoL questionnaire gave scores on a scale from 0 to 100 , where 0 represents the value of the lowest quality of life and 100 represents the highest quality of life.

\section{Translation and cultural adaptation of the CarGoQoL}

The translation and transcultural adaptation of the CarGoQoL questionnaire was carried out after an authorization of use by MAPI RESEARCH TRUST following the procedure of the translation guide of the CarGoQoL questionnaires.

The methodological process included forward and backward translation. The original version of the CarGoQoL has been translated by two independent translators from French to the Moroccan Arabic dialect. Two other translators, unfamiliar with the original version, carried out a back-translation of the synthesis of the two translated versions. The translated and back-translated versions were reviewed by a committee of experts. The final Moroccan Arabic dialect version was drafted after a pre-test in about 20 caregivers of palliative cancer patients admitted to the Rabat National Institute of Oncology and declared palliative.

\section{Subjects recruitment}

The study was conducted with a group of caregivers of palliative cancer between December 2017 and July 2018, at the national institute of oncology in RABAT. It is the first reference center for the diagnosis and treatment of cancer in Morocco.

The caregivers included were the carers of cancer patients declared palliative (out of all therapeutic resources) admitted to the national oncology institute of RABAT.

The number of subjects required was established using the Streiner curve which indicates that for a value of intra-class correlation coefficient around 0.70 and an accuracy of 0.10 , the number of subjects should be at least 120 [16].

\section{Instruments and procedures}

The Moroccan version of CarGoQoL was administered to caregivers of palliative diagnosed cancer patients. The same questionnaire was administered again from the third to the seventh day later to assess reproducibility. Participants provided socio-demographic and clinical data.

\section{Statistical analysis}

The psychometric tests were carried out according to the following procedure: Evaluate missing data and score distribution (mean, interval, floor and ceiling effects). The reliability of internal consistency was assessed by the Cronbach coefficient $\alpha$, a value of 0.70 or more was considered adequate [17]. Inter-observer reproducibility was tested using the intra-class correlation coefficient (ICC). The acceptability of the CarGoQoL was assessed with the response rate, percentage of missing data and time required to complete the questionnaire. $\mathrm{p}$ value of $<0.05$ was considered statistically significant.

Multi-treatment scale analysis was used to examine the following elements: Convergent validity and discriminant validity by determining the extent to which items are in correlation with the dimension assumed to be represented (items must have a significantly higher correlation with their own dimension than with other dimensions). The correlations of the items to their own dimensions must be $>0.40$.

The discriminant validity of CarGOQoL was assessed using the clinical and sociodemographic characteristics. All statistical analyses were performed using SPSS 13.0.

\section{Results}

120 caregivers were included in this study. Participants were aged 17 to 67 years. $86,7 \%$ were women and $45 \%$ had a secondary education. The most common primary cancer diagnoses were respectively (43\% gynecological and breast, $37.5 \%$ digestive, $22 \%$ lung). The median care giving duration was 13.06 months. Other socio-demographic and clinical characteristics are presented in (Table 1).

The average time to complete the questionnaire was 9 minutes, with missing data of $1.7 \%$ for the Social support dimension and $29.2 \%$ for the private life dimension. The floor effect ranged from $0.8 \%$ to $2.5 \%$ and the ceiling effect from $16.7 \%$ to $38.9 \%$ (Table 2).

Internal consistency for the overall index was $\alpha=0.82$. Reliability was explored using test-retest data with 20 caregivers, including CarGoQoL administered twice in a 3-7-day interval. (CCI) was from 0.76 to 0.98 . The correlation between each item and its own dimension was higher than the correlation with the other dimensions (Table 3).

Interdimensional correlations were significant $(r>0.4)$ with the exception of administration and finance (AF), self-esteem (SE) and leisure time (LEI). A high correlation was noted between the psychological dimension wellbeing (PsWB) and burden (B), coping (COP) and (PsWB), physical wellbeing (PhWB) and (COP), (B) and $(\mathrm{COP})$ and between $(\mathrm{B})$ and $(\mathrm{PhWB})$, with a range from 0.55 to 0.77 (Table 4).

The discriminating validity of CarGoQoL was assessed using sociodemographic characteristics (Table 5). Women reported statistically significant lower scores for PhWB and AF dimensions than men. A physical burden $(\mathrm{PhWB})$ was reported when the caregiver is a parent of the patient. An activity limit (LEI) was reported in the children of patients. A privacy disruption was reported when the caregiver is a 
Table 1. Caregivers sociodemographic and clinical characteristics

\begin{tabular}{|c|c|c|}
\hline Caregivers & & $\mathrm{N}=\mathbf{1 2 0}$ \\
\hline Age (Years) & Mean $\pm \mathrm{SD}^{\mathrm{a}}$ & $45.47 \pm 11.7$ \\
\hline \multirow{2}{*}{ Gender } & Women & $105(86.7)$ \\
\hline & Men & $16(13.3)$ \\
\hline \multirow{3}{*}{ Residence } & Urban & $98(81.7)$ \\
\hline & Rural & $19(15.8)$ \\
\hline & NP & $3(2.5)$ \\
\hline \multirow{5}{*}{ Relationship with the patient } & Partner & $35(29.2)$ \\
\hline & Parent & $40(33.4)$ \\
\hline & Children & $18(15)$ \\
\hline & Brother/Sister & $25(20.9)$ \\
\hline & Others $^{\mathrm{b}}$ & $2(1.6)$ \\
\hline \multirow{4}{*}{ Education } & illiterate & $25(20.8)$ \\
\hline & Primary & $41(34.2)$ \\
\hline & secondary & $45(37.5)$ \\
\hline & university & $9(7.5)$ \\
\hline \multirow{3}{*}{ Employment status } & Unemployed & $70(58.3)$ \\
\hline & Employed & $47(39.1)$ \\
\hline & NP & $3(2.5)$ \\
\hline \multirow{5}{*}{ Primary cancer diagnosis } & Lung & $22(18.3)$ \\
\hline & Digestive & $45(37.5)$ \\
\hline & Gynecological and Breast & $43(35.8)$ \\
\hline & urological & $5(4.2)$ \\
\hline & Others ${ }^{\mathrm{c}}$ & $5(4.2)$ \\
\hline \multirow{3}{*}{ Karnofsky index } & $\leq 30$ & $31(25.8)$ \\
\hline & [40- 50] & $79(65.9)$ \\
\hline & $\geq 60$ & $19(8.4)$ \\
\hline Duration of patient assistance (Months) & Median & $13.06[2-72]$ \\
\hline Time to transition to palliative care (Months) & Median & $22.89[1-204]$ \\
\hline
\end{tabular}

${ }^{a}$ SD standard deviation ${ }^{b}$ friend (1) other family member ${ }^{\mathrm{C}}$ Cavum (2) thyroid (1) unknown primary (2)

Table 2. Dimensions' characteristics of CarGoQoL

\begin{tabular}{|c|c|c|c|c|}
\hline Dimension/Item & $\mathbf{N}$ & $\mathbf{M} \pm \mathbf{S D}^{\mathrm{a}}$ & Floor effect ${ }^{b}(\%)$ & Ceiling effect ${ }^{\mathrm{c}}(\%)$ \\
\hline Psychological wellbeing (4) & 120 & $68.43 \pm 31.50$ & 0.8 & 23.3 \\
\hline Burden (4) & 120 & $66.47 \pm 29.10$ & 0.8 & 16.7 \\
\hline Relationship with healthcare (3) & 120 & $67.98 \pm 18.55$ & 0.8 & 38.9 \\
\hline Administration and finances (3) & 120 & $58.75 \pm 18.26$ & 2.5 & 29.2 \\
\hline Coping (3) & 120 & $80.06 \pm 17.22$ & 0.8 & 36.7 \\
\hline Physical wellbeing (4) & 120 & $70.64 \pm 21.87$ & 0.8 & 18.3 \\
\hline Self-esteem (2) & 120 & $24.47 \pm 19.13$ & 0.8 & 32.2 \\
\hline Leisure time (2) & 120 & $74.68 \pm 12.49$ & 0.8 & 20.8 \\
\hline Social support (2) & 118 & $71.61 \pm 21.22$ & 0.8 & 38.3 \\
\hline Privatelife (2) & 85 & $65.73 \pm 12.22$ & 0.8 & 28.8 \\
\hline
\end{tabular}

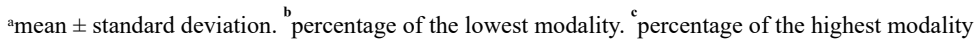

Table 3. Internal reliability and validity of the CarGoQoL

\begin{tabular}{|c|c|c|c|}
\hline & Cronbach's $\alpha$ & $\begin{array}{c}\text { IIC } \\
\text { Min-Max }\end{array}$ & $\begin{array}{c}\text { Reliability ICC } \\
\text { Test-retest }\end{array}$ \\
\hline PsWB & 0.96 & $0.89-0.94$ & $0.41-0.94$ \\
\hline B & 0.91 & $0.82-0.94$ & $0.30-0.65$ \\
\hline RHC & 0.94 & $0.91-0.95$ & $0.42-0.77$ \\
\hline AF & 0.87 & $0.69-0.92$ & $0.42-0.76$ \\
\hline COP & 0.66 & $0.66-0.87$ & 0.95 \\
\hline PhWB & 0.96 & $0.90-0.91$ & $0.48-0.76$ \\
\hline SE & 0.68 & 0.89 & $0.42-0.69$ \\
\hline SEI & 0.65 & 0.87 & 0.76 \\
\hline PL & 0.82 & 0.93 & 0.98 \\
\hline
\end{tabular}

PsWB: Psychological wellbeing; B: Burden; RHC: Relationship with healthcare; AF: Administration and finances; COP: Coping; PhWB: Physical wellbeing; SE: Self-esteem; LEI: Leisure time; SS: Social support; PL: Private life 
Table 4. Inter-dimensional correlation of the CarGoQoL

\begin{tabular}{|c|c|c|c|c|c|c|}
\hline & PsWB & B & RHC & AF & COP & PhWB \\
\hline PsWB & & & & & \\
\hline B & $0.559^{* *}$ & & & & \\
\hline RHC & $-0.248^{* *}$ & $-0.296^{* *}$ & & & \\
\hline AF & & & & & \\
\hline COP & $0.711^{* *}$ & $0.405^{* *}$ & $-0.293^{* *}$ & & \\
\hline PhWB & $0.776^{* *}$ & $0.437^{* *}$ & $-0.266^{* *}$ & & \\
\hline SE & & $0.278^{* *}$ & & & \\
\hline LEI & $-0.225^{* *}$ & & & & \\
\hline SS & $-0.212^{*}$ & $-0.296^{* *}$ & $0.202^{*}$ & $-0.180^{* *}$ & \\
\hline PL & $0.219^{*}$ & $0.348^{* *}$ & & $-0.277^{* *}$ & $-0.204^{*}$ & $-0.431^{* *}$ \\
\hline
\end{tabular}

$\mathrm{p}<0.05, * * \mathrm{p}<0.01, * * * \mathrm{p}<0.001$

Table 5. Comparisons (mean \pm standard deviation) and correlations of CarGOQoL scores with respect to caregivers' and patients' characteristics

\begin{tabular}{|c|c|c|c|c|c|c|c|c|c|c|}
\hline & PsWB & B & RHC & AF & COP & PhWB & SE & LEI & SS & PL \\
\hline \multicolumn{11}{|c|}{ Caregiver's gender } \\
\hline women & $68.7 \pm 31.7$ & $68.4 \pm 28,2$ & $67.1 \pm 18.6$ & $57.2 \pm 17.1$ & $80.3 \pm 16.9$ & $70.1 \pm 21.7$ & $25.0 \pm 19.2$ & $75.2 \pm 12.5$ & $71.7 \pm 20.8$ & $66.1 \pm 12.1$ \\
\hline men & $66.5 \pm 31.02$ & $53.7 \pm 32,5$ & $73.4 \pm 17.8$ & $68.7 \pm 22.2$ & $78.1 \pm 19.6$ & $73.8 \pm 22.8$ & $21.0 \pm 18.6$ & $71.0 \pm 11.8$ & $70.5 \pm 24.8$ & $63.4 \pm 12.9$ \\
\hline$P$ value & 0.8 & 0.04 & 0.2 & 0.01 & 0.6 & 0.03 & 0.4 & 0.2 & 0.8 & 0.4 \\
\hline \multicolumn{11}{|c|}{ Relationship status } \\
\hline Partner & $58.8 \pm 36.7$ & $64.4 \pm 30.1$ & $64.7 \pm 17.1$ & $60.2 \pm 20.9$ & $76.4 \pm 17.2$ & $57.3 \pm 26.3$ & $17.5 \pm 15.2$ & $81.4 \pm 11.8$ & $72.1 \pm 19.4$ & $59.6 \pm 10.0$ \\
\hline Parent & $72.7 \pm 30.3$ & $71.2 \pm 29.2$ & $64.1 \pm 19.9$ & $56.2 \pm 17.0$ & $81.0 \pm 18.3$ & $77.6 \pm 17.2$ & $27.5 \pm 20.8$ & $73.1 \pm 10.4$ & $70.3 \pm 22.5$ & $70.8 \pm 10.2$ \\
\hline Child & $65.7 \pm 29.7$ & $69.2 \pm 30.5$ & $73.6 \pm 21.0$ & $66.2 \pm 16.2$ & $76.8 \pm 19.0$ & $66.6 \pm 18.6$ & $30.5 \pm 24.7$ & $69.4 \pm 17.7$ & $66.6 \pm 26.4$ & $68.7 \pm 13.5$ \\
\hline Brother/sister & $75.2 \pm 24.7$ & $58.6 \pm 27.1$ & $75.0 \pm 14.0$ & $55.3 \pm 17.3$ & $85.0 \pm 13.3$ & $80.0 \pm 14.3$ & $24.0 \pm 14.3$ & $71.5 \pm 8.4$ & $76.6 \pm 17.7$ & $67.1 \pm 14.3$ \\
\hline$P$ value & 0.1 & 0.4 & 0.08 & 0.3 & 0.2 & 0.00 & 0.07 & 0.003 & 0.6 & 0.009 \\
\hline \multicolumn{11}{|c|}{ Caregiver's age class } \\
\hline$<40$ & $51.9 \pm 30.1$ & $48.1 \pm 27.4$ & $74.5 \pm 20.5$ & $55.1 \pm 16.4$ & $74.2 \pm 18.3$ & $61.8 \pm 22.1$ & $20.9 \pm 18.1$ & $76.1 \pm 13.8$ & $78.7 \pm 17.2$ & $62.5 \pm 9.4$ \\
\hline $40-60$ & $72.4 \pm 30.3$ & $70.4 \pm 25.4$ & $67.3 \pm 16.8$ & $61.0 \pm 18.7$ & $81.2 \pm 17.0$ & $71.6 \pm 20.5$ & $27.4 \pm 19.4$ & $11.9 \pm 1.4$ & $71.2 \pm 21.6$ & $66.4 \pm 13.1$ \\
\hline$>60$ & $86.7 \pm 24.1$ & $89.1 \pm 27.0$ & $56.1 \pm 16.2$ & $56.1 \pm 19.2$ & $87.7 \pm 10.8$ & $85.8 \pm 18.6$ & $18.3 \pm 18.2$ & $75.0 \pm 12.5$ & $57.5 \pm 21.0$ & $66.3 \pm 10.6$ \\
\hline$P$ value & 0.00 & 0.00 & 0.005 & 0.2 & 0.02 & 0.001 & 0.1 & 0.7 & 0.005 & 0.5 \\
\hline \multicolumn{11}{|c|}{ Patient's gender } \\
\hline Women & $69.7 \pm 29.5$ & $65.0 \pm 28.0$ & $71.0 \pm 18.8$ & $58.0 \pm 16.4$ & $80.0 \pm 17.8$ & $74.2 \pm 18.2$ & $24.2 \pm 20.1$ & $73.3 \pm 10.7$ & $74.2 \pm 21.8$ & $68.3 \pm 11.3$ \\
\hline Men & $67.4 \pm 34.3$ & $68.0 \pm 30.8$ & $63.5 \pm 17.7$ & $60.1 \pm 20.7$ & $80.6 \pm 15.8$ & $65.5 \pm 25.2$ & $25.0 \pm 18.2$ & $76.5 \pm 14.6$ & $67.8 \pm 19.7$ & $62.8 \pm 12.8$ \\
\hline$P$ value & 0.3 & 0.7 & 0.08 & 0.5 & 0.08 & 0.06 & 0.9 & 0.4 & 0.1 & 0.1 \\
\hline \multicolumn{11}{|c|}{ Patient' age class(years) } \\
\hline$<30$ & $54.5 \pm 39.2$ & $78.6 \pm 35.4$ & $70.2 \pm 23.0$ & $52.3 \pm 12.4$ & $60.7 \pm 26.2$ & $60.7 \pm 27.4$ & $42.8 \pm 32.1$ & $83.9 \pm 9.4$ & $64.2 \pm 31.8$ & $70.0 \pm 6.8$ \\
\hline $30-70$ & $60.7 \pm 33.0$ & $58.0 \pm 28.5$ & $69.8 \pm 17.1$ & $57.5 \pm 17.7$ & $77.5 \pm 17.1$ & $65.4 \pm 22.2$ & $22.6 \pm 17.2$ & $75.5 \pm 12.9$ & $76,1 \pm 16.4$ & $64.0 \pm 12.6$ \\
\hline$>70$ & $86.1 \pm 16.7$ & $80.9 \pm 22.2$ & $63.8 \pm 20.2$ & $62.2 \pm 19.9$ & $88.5 \pm 9.7$ & $82.7 \pm 14.1$ & $24.6 \pm 18.4$ & $71.3 \pm 11.2$ & $63.8 \pm 25.1$ & $67.4 \pm 12.0$ \\
\hline$P$ value & $\mathbf{0 . 0 0}$ & 0.00 & 0.2 & 0.2 & 0.00 & 0.00 & 0.02 & 0.03 & 0.009 & 0.3 \\
\hline \multicolumn{11}{|c|}{ Caregiving duration (months) } \\
\hline$<6$ & $75.5 \pm 29.3$ & $76.3 \pm 26.3$ & $67.0 \pm 18.2$ & $60.8 \pm 21.2$ & $82.9 \pm 17.3$ & $77.4 \pm 17.5$ & $35.8 \pm 21.7$ & $73.9 \pm 11.2$ & $65.9 \pm 27.3$ & $71.8 \pm 11.6$ \\
\hline $6-24$ & $65.3 \pm 32.2$ & $64.8 \pm 28.4$ & $68.3 \pm 19.2$ & $56.8 \pm 16.7$ & $78.5 \pm 17.8$ & $68.2 \pm 22.9$ & $22.5 \pm 17.5$ & $74.8 \pm 13.3$ & $72.3 \pm 19.3$ & $63.9 \pm 11.6$ \\
\hline$>24$ & $77.0 \pm 29.2$ & $57.1 \pm 38.1$ & $65.0 \pm 12.9$ & $71.6 \pm 20.1$ & $85.0 \pm 10,2$ & $73.7 \pm 19.0$ & $12.5 \pm 13.1$ & $73.7 \pm 7.0$ & $77.5 \pm 21.0$ & $67.1 \pm 14.8$ \\
\hline $\mathrm{p}$ value & 0.2 & 0.1 & 0.8 & 0.04 & 0.3 & 0.1 & 0.001 & 0.9 & 0.2 & 0.06 \\
\hline \multicolumn{11}{|c|}{ Patient's disease duration (months) } \\
\hline$<12$ & $68.6 \pm 32.6$ & $71.2 \pm 28.1$ & $68.0 \pm 19.9$ & $60.7 \pm 18.7$ & $80.3 \pm 18.1$ & $70.6 \pm 21.5$ & $27.5 \pm 21.3$ & $74.5 \pm 11.5$ & $69.1 \pm 22.3$ & $68.7 \pm 11.3$ \\
\hline $12-48$ & $65.3 \pm 32.4$ & $62.5 \pm 31.7$ & $65.3 \pm 16.9$ & $57.5 \pm 17.8$ & $78.8 \pm 17.5$ & $67.3 \pm 24.3$ & $20.9 \pm 16.7$ & $77.9 \pm 11.8$ & $72.0 \pm 21.2$ & $61.4 \pm 11.8$ \\
\hline$>48$ & $72.9 \pm 27.0$ & $55.9 \pm 24.0$ & $75.5 \pm 17.9$ & $51.6 \pm 16.1$ & $80.5 \pm 14.3$ & $78.7 \pm 15.4$ & $20.0 \pm 13.1$ & $68.3 \pm 14.8$ & $79.4 \pm 14.3$ & $67.8 \pm 15.9$ \\
\hline$P$ value & 0.7 & 0.1 & 0.1 & 0.2 & 0.9 & 0.2 & 0.14 & 0.03 & 0.2 & 0.03 \\
\hline
\end{tabular}

sibling of the patient. the least senior caregivers reported a poor quality of life for the dimensions PsWB, Burden, COP and PhWB. Overall, no difference was found with respect to the patient's gender. Caregivers reported a worse quality of life in terms of emotional dimensions: PsWB, COP and physical dimension (PhWB) when the patient is younger. When the patient is older, caregivers reported lower quality of life scores in dimensions B, SE, LEI and SS. Caregivers reported a lower quality of life for the AF and SE dimensions when the duration of assistance increases and for the LEI and PL dimensions when the duration of the illness is long.

\section{Discussion}

In this article, we present the results of a transcultural validation of the Moroccan version of CarGoQoL. This questionnaire is well designed and validated to measure and evaluate the quality of life of caregivers of cancer patients. it was developed by a French research team and has an American version conducted in the United States [10,11].

For the development of our Moroccan Arabic version; we followed a rigorous scientific methodology recommended in the field $[13,16,17]$. 
The CarGoQoL questionnaire is exclusive in identifying dimensions specific to carers.

The cross-cultural adaptation process was carried out in close collaboration with the Mapi Research Trust team that granted the conduct of this study.

The validation was conducted with a diverse sample including spouses, parents, children, siblings to identify the different links with the palliative cancer patient and covers all aspects of caregivers' quality of life.

The psychometric properties of the Moroccan Arabic version of CarGoQoL are considered satisfactory; the average time required to complete the questionnaire was similar to that of the two French and American validation studies $[10,11]$.

Only one dimension of privacy that is unsatisfactory in terms of its Internal consistency $(\alpha=0.14)$ and missing data rate $29.2 \%$, this is almost similar to the American study [11].

The test-retest time was three to seven days, this meets the recommended guidelines suggesting that the retest interval of 2 to 10 days is usually used [17].

In our study, there were more women than male caregivers, this is similar in the French and American study [10,11]. In Morocco, it is not surprising that it is women who assume the role of primary caregiver because in our culture they are often expected to be the caregiver.

In our study, caregivers experienced a ripple effect and physical burden when they are the parents, this was also in other contexts [1821].

All items were strongly correlated with their own dimensions than with others. Discriminant validity was determined between certain caregiver characteristics and quality of life scores. Previous studies demonstrated that the characteristics of patients impose a significant impact on the need for family care and the level of emotional distress of the family caregivers [19-21].

Our study confirmed that women had lower quality of life scores than men, this was strongly studied and confirmed in the literature $[22,23]$.

A strong correlation has been demonstrated in our study between the dimensions psychological well-being and physical well-being. This could be justified by the fact that psychological problems have an impact on the caregiver's physical state leading to significant physiological changes.

Also, meanings have been noted in the administrative and financial dimension among female caregiver's and when the duration of the assistance exceeds 24 months, it is explained by the material and moral commitment towards the patient, this has also been demonstrated by a study carried out in Morocco; in most cases, it is the family that assists, materially and morally helps the patient [24].

Some limitations should be taken into account: the failure to carry out external validation on a similar scale given its unavailability. The CarGoQoL is a self-administered questionnaire, but the problem of the low literacy in Morocco yielded to a self-administration or administration by the same investigator following the level of education of the patients.

Our version is in Moroccan Arabic who is the language spoken by the majority of Moroccans; but the cultural diversity in Morocco emerges from regional languages such as "Tarifit", "Tamazight" and "Tachelhit", which must be taken into account in subsequent studies.

\section{Conclusion}

The Moroccan Arabic version of CarGOQoL is a useful, reliable and valid tool to measure the quality of life of carers of palliative cancer patients in Morocco. Having a Moroccan version of CarGoQoL will make it easier for researchers to choose valid instruments to conduct studies that focus on carers.

\section{Declaration}

\section{Ethics approval and consent to participate}

All participants signed a written consent. The study has been approved by the ethics committee for biomedical research of the MOHAMED V Faculty of Medicine and Pharmacy in RABAT (N/R: folder number 15/18).

\section{Consent for publication}

Not applicable.

\section{Availability of data and materials}

All data generated or analyzed during this study are included in this published article.

\section{Competing interests} study.

The authors declare that they have no competing interests for this

\section{Funding}

There was no funding for this study.

\section{Authors' contributions}

AA designed the study and analyzed the data and wrote the manuscript. B. HR was involved in the translation and critical revision of the manuscript. RR contributed to the design, analysis, and interpretation of the data and was involved in the writing of the manuscript. EH and $\mathbf{M H}$ were critically involved in the revision of the manuscript and gave final approval of the version to be published. All authors read and approved the manuscript.

\section{Acknowledgment}

The authors thank the team of Mapi research for permitting the use of CarQoQoL. We also thank the staff at the National Oncology Institute in Rabat, Morocco for this study. We thank Ms FOUZIA HAMMOUCH, Dr LAILA LAHLOU and professor SABER BOUTAYEB for their collaboration all along the completion of this study. We thank the patients and families for their cooperation.

\section{References}

1. Bergwelt-Baildon MV, Hallek MJ, Shimabukuro-Vornhagen AA, Kochanek M (2010) CCC meets ICU. Redefining the role of critical care of cancer patients. BMC Cancer 10: 612. [Crossref]

2. Stenberg U, Ruland CM, Miaskowski C (2009) Review of the literature on the effects of caring for a patient with cancer. Psycho-Oncology 19: 1013-1025. [Crossref]

3. Lengacher CA, Kip KE, Barta M, Post-White J, Jacobsen PB, et al. (2012) A pilot study evaluating the effect of mindfulness-based stress reduction on psychological status, physical status, salivary cortisol, and interleukin- 6 among advanced-stage cancer patients and their caregivers. J Holist Nurs 30: 170-185. [Crossref] 
4. Given BA, Given CW, Kozachik S (2001) Family support in advanced cancer. $C A$ Cancer J Clin 51: 213-231.

5. Molassiotis A, Wilson B, Blair S, Howe T, Cavet J (2011) Living with multiple myeloma: experiences of patients and their informal caregivers. Support Care Cancer 19: 101-111. [Crossref]

6. Ellis J (2012) The impact of lung cancer on patients and carers. Chronic Respir Dis 95: 39-47. [Crossref]

7. Minaya Flores P, Berbis J, ChinotO, Auquier P (2014) Assessing the quality of life among caregivers of patients with glioma. Neuro Oncol Pract 1:191-197. [Crossref]

8. El -Jawahri AR, Traeger LN, Kuzmuk K, Eusebio JR, Vandusen HB, et al. (2015) Quality of life and mood of patients and family caregivers during hospitalization for hematopoietic stem cell transplantation. Cancer 121: 951-959. [Crossref]

9. Siegel K, Reveis VH, Houts P, Mor V (1991) Caregiver burden and unmet patient needs. Cancer 68: 1131-1140. [Crossref]

10. Minaya P, Baumstarck K, Berbis J, Concalves A, Barlesi F, et al. (2012) The caregiver oncology quality of life questionnaire (CarGoQoL): Development and validation of an instrument to measure the quality of life of the caregivers patients with cancer. Eur $J$ Cancer 48: 904-911. [Crossref]

11. Kaveney S, Baumstarck K, Minaya P, Shannon T, Symes P, et al. (2016) Validation of the American version of the Caregiver Oncology Quality of life (CarGoQoL) questionnaire. Health Qual Life Outcomes 14: 82. [Crossref]

12. Nejjari C, El Fakir S, Bendahhou K, El Rhazi K, Abda N, Zidouh A, et al. (2014) Translation and validation of European organization for research and treatment of cancer quality of life Questionnaire -C30 into Moroccan version for cancer patients in Morocco. BMC Research 7: 228. [Crossref]

13. Guillemin F, Bombardier C, Beaton D (1993) Cross-cultural adaptation of healthrelated quality of life measures: literature review and proposed guidelines. $J$ Clin Epidemiol 46: 1417-1432. [Crossref]
14. Lohr KN, Aaronson NK, Alomso J, Burnam MA, Patrick DL, et al. (1996) Evaluating quality-of-life and health-status instrument: development of scientific review criteria. Clin Ther 18: 979-992. [Crossref]

15. Acquadro C, Conway K, Giroudet C, Mear I (2012) Linguistic validation manual for health outcome assessments. Mapi.

16. Streiner DL, Geoffrey RN (2005) Health measurement scales. A pratical guide to their development and use 2005. 3rd edition. ISBN 978-0-19-968521-9.

17. Cronbach LJ (1951) Coeffcient alpha and the internal structure of tests. Psychometrika 16: 297-334.

18. Lund L, Ross L, Petersen MA, Groenvold M (2014) Cancer caregiving tasks and consequences and their associations with caregiver status and the caregiver's relationship to the patient: a survey. BMC Cancer 14: 541. [Crossref]

19. Northouse L, Williams AL, Given B, McCorkle R (2012) Psychosocial care for family caregivers of patients with cancer. J Clin Oncol 30: 1227-1234. [Crossref]

20. Yu H, Li L, Liu C, Huang W, Zhou J, et al. (2017) Factors associated with the quality of life of family caregivers for leukemia patients in China. Health Qual Life Outcomes 15: 55. [Crossref]

21. Awadalla W, Ohaeri JU, Gholoum A, Khalid OA, Hamad MA, et al. (2007) Factor associated with quality of life of outpatients with breast cancer and gynecologic cancers and their family caregivers: a controlled study. BMC Cancer 7: 102.

22. Janda M, Steginga S, Langbecker D, Dunn J, Walker D, et al. (2007) Quality of life among patients with a brain tumor and their carers. J Psychosom Res 63: 617-623. [Crossref]

23. Parvataneni R, Polley MY, Freeman T, Lamborn K, Prados M, et al. (2011) Identifying the needs of brain tumor patients and their caregivers. J Neurooncol 104: 737-744. [Crossref]

24. Errihani H, Abarrou N, Ayemou A, el Mesbahi O, el Mazghi A, et al. (2005) Caractéristiques psychosociales des patients cancéreux marocains: étude de 1000 cas recrutés à l'Institut national d'oncologie de Rabat. Rev Francoph Psycho-Oncologie.

Copyright: (C2021 Aitouma A. This is an open-access article distributed under the terms of the Creative Commons Attribution License, which permits unrestricted use, distribution, and reproduction in any medium, provided the original author and source are credited. 